\title{
Sur la grandeur relative des infinis des fonctions.
}

\author{
(par Paul du Bois-Reymond, professeur $\dot{a}$ l'Université \\ de Freiburg en Bade.)
}

On s'occupera dans ce petit mémoire de la limite du rapport de deux fonctions $f(x)$ et $\varphi(x)$, ces fonctions devenant infinies ou s'annulant pour $x=\infty$. Notre but ne sera pas d'établir la valeur finie, quand elle existe, de la limite ou rapport $\frac{f(x)}{\varphi(x)}$. Mais nous nous proposons premièrement de développer quelques vues générales, d'ailleurs en partie connues, concernant la suite continue des fonctions ordonnées suivant les limites de leurs quotients et l'analogie de cette suite avec la suite des nombres réels (art. I), et secondement (art. II) de démontrer certains théorèmes, qui peuvent servir à classer les fonctions selon la limite du rapport $\frac{d \log f(x)}{d x}$, et qui, en déterminant dans un grand nombre de cas la vitesse avec laquelle la dérivée s'approche de l'infini, lorsque celle de la fonction primitive est donnée, pourront être utiles dans la théorie de la convergence des integrales: savoir quand, la fonction étant donnée en série, il n'est pas permis de la différentier membre à membre, ou quand la fonction n'est connue que par certaines propriétés suffisantes pour la solution du problème $\left(^{*}\right)$.

Pour plus de concision dans l'énoncé des théorèmes et dans les démonstrations nous nous servirons des expressions et des notations suivantes:

S'il s'agit de comparer deux fonctions d'une variable $x$ par rapport à la vitesse avec laquelle elles atteignent l'infini, $x$ devenant infinie, il est clair

(*) Le rôle que la dérivée joue dans la théorie de la convergence des intégrales se trollvera exposé dans un petit ouvrage de l'auteur sur la convergence et la divergence des intégrales et des series, qui va paraître chez B. G. Teubner, Leipzig. 
qu'une fonction $f(x)$ rapprochera infiniment plus vite ou infiniment moins vite de sa limite infinie, qu'une autre fonction $\varphi(x)$, suivant que le rapport $\frac{f(x)}{\rho(x)}$ tendra vers une limite infinie ou s'annulera, pendant que $x$ croit à l'infini. Quand $\lim \frac{f(x)}{\varphi(x)}$ est infinie, nous dirons que $f(x)$ a un infini plus grand que $\varphi(x)$, expression pas assez philosophique, j'en conviens, mais qui a l'avantage d'être brève et claire. De même lorsque $\lim \frac{f(x)}{\varphi(x)}$ est finie ou est égale à zéro, nous dirons que $f(x)$ a un infini égal ou inférieur à celui de $\varphi(x)$. Et nous ferons usage des notations:

$$
f(x)>\varphi(x), \quad f(x) \sim \varphi(x), \quad f(x)<\varphi(x)
$$

qui seront équivalentes à ces formules:

$$
\lim \frac{f(x)}{\varphi(x)}=\infty, \quad \lim \frac{f(x)}{\varphi(x)} \text { est finie, } \lim \frac{f(x)}{\varphi(x)}=0 .
$$

Ce nouvel algorithme, qui présente quelque analogie avec les inégalités ordinaires, pourra être nommé celui des inégalités infinitaires. Soient donnés par exemple trois couples de fonctions satisfaisantes aux inégalités infinitaires :

$$
f(x)>f_{1}(x), \quad \phi(x)>\varphi_{1}(x), \quad \lambda(x) \sim \lambda_{1}(x)
$$

on aura aussi :

$$
f(x) \varphi(x)>f_{1}(x) \phi_{1}(x), \quad f(x) \lambda(x)^{ \pm 1}>f_{1}(x) \lambda_{1}(x)^{ \pm 1},
$$

et, en cas que $f(x)>\lambda(x)$, on aura $f(x) \pm \lambda(x)>f_{1}(x) \pm \lambda_{1}(x)$, mais, si $\lambda(x)>$ $f(x)$, l'analogie fait défaut, et on aura $f(x) \pm \lambda(x) \backsim f_{1}(x) \pm \lambda_{1}(x)$, formules qu'il est aisé de vérifier.

Comme ces inégalités n'expriment que des propriétés de la limite du quotient de deux fonctions, on peut s'en servir encore quand ces fonctions s'évanouissent à la limite, au lieu de devenir infinies. Or si deux fonctions s'évanouissent pour $x=\infty$, pourquoi ne pas attribuer aussi à zéro, symboliquement parlant, des grandeurs différentes et comparables, et dire que $f(x)$ a un zéro égal à celui de $\phi(x)$, ou bien plus grand ou plus petit que celui de $\varphi(x)$, selon qu'on trouve $f(x) \smile \varphi(x), f(x)>\varphi(x), f(x)\langle\varphi(x)$ ? 


\section{Art. I.}

1. Suite des fonctions ordonnée suivant les valeurs limites de leurs quotiens.

Les fonctions rangées par ordre de grandeur de leurs infinis forment une suite infinie. Pour s'en faire une idée, on commencera par ordonner en série, suivant leurs infinis les fonctions, les plus usitées:

$$
f_{0}(x) \ldots f_{1}(x) \ldots f_{2}(x) \ldots,
$$

de manière que chaque fonction suivante ait un infini plus grand que toutes les précédentes. Qu'il s'agisse de placer dans cette série une fonction donnée $\psi(x)$, on y cherchera deux fonctions $f_{n-1}(x)$ et $f_{n}(x)$, telles qu'on ait: $f_{n-1}<\psi<f_{n}$, et on placera $\psi$ entre $f_{n-1}$ et $f_{n}$. Faisant

$$
\log \text { nat } x=1 x, \quad 11 x=l_{2} x, \quad 111 x=l_{3} x \text {, etc. }
$$

une pareille série sera:

$$
\begin{array}{r}
\ldots e^{-\mu e^{\mu_{1} x^{x}}} \ldots e^{-\mu x^{x}} \ldots x^{-\mu} \ldots(1 x)^{-\mu} \ldots\left(1_{2} x\right)^{-\mu} \ldots\left(1_{3} x\right)^{-\mu} \ldots \text { const } \ldots\left(1_{3} x\right)^{\mu} \\
\ldots\left(1_{2} x\right)^{\mu} \ldots(1 x)^{\mu} \ldots x^{\mu} \ldots e^{\mu x^{x}} \ldots e^{\mu e^{\mu_{1} x^{\alpha}}} \ldots .
\end{array}
$$

où à gauche de «const. 》 les fonctions sont ordonnées suivant la grandeur des infinis de leur valeurs reciproques, à droite suivant les infinis de leurs valeurs directes, en sorte que le quotient d'une fonction quelconque de la série par une précédente devient infini pour $x=\infty$, ce dont on se convaincra aisément dans la plupart des cas, en ramenant ces quotients à la forme $\frac{x}{1 x}$, ainsi que je le ferai voir par quelques exemples. Cherchons d'abord la limite de $\frac{x^{\mu}}{(1 x)^{m}}$. On a $\frac{x^{\mu}}{(1 x)^{n}}=\left(\frac{x^{\frac{\mu}{m}}}{1 x}\right)^{m}$. En posant $x^{\frac{\mu}{m}}=x_{1}$, on trouve $\lim \frac{x^{\mu}}{(1 x)^{m}}=\frac{1}{\left(\frac{m}{\mu}\right)^{m}} \lim \left(\frac{x_{1}}{\left(x_{1}\right.}\right)^{m}=\infty$. Établissons encore la limite de $x^{-m} e^{\mu x^{x}}$. Posant $\mu x^{x}=x_{1}$, cette expression devient $\mu^{\frac{m}{x}} \cdot x_{1}^{-\frac{m}{x}} e^{x_{1}}$, dont le 
logarithme peut être mis sous la forme: $1 \mu^{\frac{m}{x}}+1 x_{1}\left\{\frac{x_{1}}{1 x_{1}}-\frac{m}{x}\right\}$. Cherchons enfin la limite de $e^{\mu_{1} x^{x_{1}}} \cdot e^{-\mu x^{x}}$, dont le logarithme est $\mu x^{x}\left\{\frac{\mu_{1}}{\mu} x^{x_{1}-x}-1\right\}$. La limite de cette expression est infinie pour $x_{1} \geqq x, \mu_{1} \geqq \mu$, excepté le cas où l'on a à la fois $x_{1}=x, \mu_{1}=\mu$.

Il y a quelques remarques à faire sur la série $F$, telle que nous l'avons écrite.

1.) Chaque membre de la série $F$ est affecté d'un ou de plusieurs paramètres $\mu, \mu_{1}, \ldots x, \ldots$ Il faut se représenter ces paramètres variant de 0 excl. jusq'à $\infty$ excl., ce qui fait que chaque fonction de la série pourvue d'un paramètre représente un infinité simple de fonctions, chaque fonction munie de deux paramètres représente une infinité double de fonctions, etc.

2.) Le produit d'un nombre fini quelconque des fonctions constituant la suite $F$, telle que nous l'avons écrite, et pour des valeurs quelconques des paramètres qu'elles contiennent, a un infini moindre que chaque fonction située à droite des fonctions, dont est composé le produit. Par exemple on a:

$$
\left(1_{2} x\right)^{m_{2}}\left(1_{3} x\right)^{m_{3}} \ldots\left(1_{n} x\right)^{m_{n}}<(1 x)^{\mu^{\mu}}
$$

quelque grands que soient les nombres $m_{2}, m_{3}, \ldots m_{n}$, et quelque petit que soit $\mu$. Cette remarque pourra servir à multiplier indéfiniment les fonctions de la série $F$. Car, par exemple, entre les fonctions $(1 x)^{\mu},(1 x)^{\mu_{1}}, \mu_{1}-\mu$ étant d'une petitesse quelconque, on pourra interposer

$$
(1 x)^{\mu}\left(1_{2} x\right)^{m_{9}}\left(1_{3} x\right)^{m_{3}} \ldots\left(1_{n} x\right)^{n_{n}}
$$

et faire varier les $m$ de 0 excl. à $\infty$ excl.

En général, soient $u_{1}, u_{2}$ deux fonctions quelconques pour lesquelles on aie $u_{1} \prec u_{2}$, en posant $u_{2}=u_{1} \varphi(x)$, on aura $\varphi(\infty)=\infty$, et encore :

$$
u_{1}<u_{1} \varphi(x)^{\mu}(1 \varphi)^{m_{1}}\left(1 _ { 2 } \varphi ( x ) ^ { m _ { 1 } } \ldots \left(1_{n} \varphi(x)^{m_{m}}<u_{2},\right.\right.
$$

$\mu$ étant $<1$.

3.) Il va sans dire, qu'il existe une foule de procédés servant à rendre plus dense la suite $F$. Notons encore celui-ci. En substituant aux paramètres $\mu$, etc. des fonctions convenablement choisies, on obtiendra autant de fonctions intermédiaires aux fonctions de la série, qu'on voudra. Ainsi 
par exemple on a:

$$
\begin{aligned}
& (1 x)^{M}<x^{\frac{1}{(1 x)^{\mu .}}}<x^{m}, \quad 0<\mu<1, \\
& (1 x)^{M}<(1 x)^{(l x)^{\mu}}<x^{m}, \quad 0<\mu<1,
\end{aligned}
$$

quelque grand que soit $M$ et quelque petit que soit $m$. Pour comparer les termes moyens de ces deux inégalités infinitaires, à cause de $x=(1 x)^{\frac{1 x}{\lg _{2} x}}$, on pourra écrire:

d'où l'on tire:

$$
(1 x)^{-(1 x)^{\mu}} x^{\frac{1}{(1 x)^{\mu_{1}}}}=1 x^{\frac{(1 x)^{1}-\mu_{1}}{l_{2} x}-(1 x)^{\mu k}}
$$

$$
(1 x)^{\left(1 x^{\mu}\right.} \succ x^{\frac{1}{\left(\mid x_{i}^{\mu}\right.}}
$$

selon que $\mu+\mu_{1}<1$ ou $\equiv 1$. Jamais on a $(1 x)^{(1 x)^{\mu}} \sim x^{\frac{1}{\left(1 x^{\mu_{1}}\right.}}$.

Pour obtenir de nouvelles fonctions intermediaires, on fera varier $\mu$ dans $(1 x)^{(1 x)^{\mu}}$ ou dans $x^{\frac{1}{(1 x)^{\mu}}}$, etc. etc.

4.) Dans le logarithme multiple $]_{n} x$, l'index $n$ pourra être choisi aussi grand qu'on voudra, mais il n'est pas permis de le faire croitre indefiniment, sans que $1_{n} x$ ne cesse d'être réel, ce qui devient évident en construisant le logarithme $1_{12} x$.

Tracons pour cet effet dans le plan des $x$ et des $y$ les lignes:

$$
y=x, \quad y=1 x \text {. }
$$

Si du point $x=X, y=1 X$ on tire une parallele à l'axe des $x$, jusqu'à la ligne $y=x$, qu'elle coupera au point $x=1 X, y=1 X$, la perpendiculaire à l'axe des $x$ passant par ce dernier point coupera la ligne $x=1 x$ au point $x=1 X, y=\mathrm{l}_{2} X$. De la même manière on construira d'abord le point $x=\mathrm{l}_{2} X$, $y=1_{2} X$, puis le point $x=1_{2} X, y=1_{3} X$, et ainsi de suite. Il est clair que, quelque grand que soit $X$, si l'on fait croitre indéfiniment $n, 1_{n} X$ finira toujours par obtenir une valeur negative, en sorte que $1_{n+1} X$ deviendra imaginaire. 
2. Analogie de la suite $F$ avec la suite des nombres réels. Cette analogie, qui est defectueuse sous un certain rapport, peut être rendue parfaite.

Comme entre deux fonctions, aussi proches par rapport à leurs infinis qu'on voudra, on peut en imaginer une infinité d'autres formant une espèce de passage de la première fonction à la seconde, on a comparé la suite $F$ à la suite des nombres réels, dans laquelle on peut aussi passer d'un nombre à un nombre très peu different par une infinité d'autres. Mais il y a entre les deux suites cette différence importante, que dans la suite des nombres, quelque serrée qu'on la suppose, on peut assigner à chaque nouveau nombre donné une place absolument déterminée, tandis que la place que devra occuper dans la suite $F$ une fonction donnée restera jusqu'à un certain point arbitraire, parce que pour chaque fonction il en a une quantité illimitée d'autres qui ont un infini égal. En effet soient $f, f_{1}, f_{2}, \ldots$ des fonctions satisfaisant à la condition:

$$
f>f_{1}>f_{2} \ldots
$$

on aura toujours:

$$
f \sim \alpha f+\alpha_{1} f_{1}+\alpha_{2} f_{2}+\cdots
$$

les $\alpha$ étant des constantes quelconques. Par exemple on voit sur le champ que

$$
x+x^{\frac{1}{2}}, \quad x \frac{x+1}{x-1}, \frac{1}{\log \left(1+\frac{1}{x}\right)}
$$

sont trois fonctions à infinis égaux.

Ici donc l'analogie entre la suite $F$ et la suite des nombres se trouve être en défaut, mais je vais montrer, comment on réussit à la rendre parfaite, moyennant quelques restrictions concernant les fonctions à admettre dans la série.

Nous y parviendrons, en ne nous bornant pas à considérer la limite du rapport $\frac{f(x)}{\varphi(x)}$, mais en éxaminant encore la différence $f(x)-\phi(x)$. Par rapport à cette différence deux choses peuvent avoir lieu. Premièrement, $x$ croissant indéfiniment, la différence $f(x)-\varphi(x)$ pourra finir par ne plus changer de signe. Secondement, cette différence pourra ne pas cesser de 
changer de signe; en d'autres termes, l'équation $f(x)-\varphi(x)=0$ pourra avoir un nombre infini de racines. Le dernier cas pourra avoir lieu quand même les deux courbes $y=f(x), y=\Phi(x)$ sont privées de points d'inflexion, comme pour

$$
f(x)=x^{2}+\sin x, \phi(x)=x^{2}
$$

Il n'aura pas lieu, si les fonctions $f(x)$ et $\Phi(x)$ sont composées algébriquement de puissances, racines, exponentielles, et opérations pareilles. Nous supposerons que parmi les fonctions, que nous considérons, il ne s'en trouvera pas deux, dont la différence, égalée a zéro, ait un nombre infini de racines. Cela posé, l'incertitude touchant l'ordre des places, qu'occuperont deux fonctions à infinis égaux dans la série, cessera, si l'on établit la règle suivante:

Une fonction $f_{2}(x)$ devra être placée à droite d'une autre fonction $f_{1}(x)$, si la différence $f_{2}(x)-f_{1}(x), x$ croissant à l'infini, finit par rester positive.

Il est nécessaire qu'elle finisse par rester ou positive ou négative, car si elle restait zéro, les deux fonctions seraient identiques. Le quotient $\frac{f(x)}{\varphi(x)}$ étant plus grand ou moins grand que l'unité, selon que la différence $f(x)-\varphi(x)$ est positive ou négative, il existe entre le premier procédé pour constituer les fonctions en série et celui que nous venons d'exposer ce rapport, qu'au lieu d'examiner simplement si la limite $\lim _{x=\infty} \frac{f(x)}{p(x)}$ est infinie ou zéro; on se demande si elle est plus grande ou plus petite que l'unité, de sorte que le premier procédé est contenu dans le second.

Appliquons le second procédé aux fonctions

$$
x+x^{\frac{1}{2}}, \quad x \frac{x+1}{x-1}, \quad \log ^{-1}\left(1+\frac{1}{x}\right) .
$$

D'abord on a:

$$
x+x^{\frac{1}{2}}-x \frac{x+1}{x-1}=\frac{x^{\frac{3}{3}}}{x-1}\left\{1-\frac{2}{x^{\frac{1}{2}}}-\frac{1}{x}\right\}
$$

différence qui finit par ètre positive. La différence $\log ^{-1}\left(1+\frac{1}{x}\right)-x \frac{x+1}{x-1}$ peut être mise sous la forme:

$$
\frac{u e^{u}+\left(1-e^{u}\right)\left(2-e^{u}\right)}{u\left(1-e^{u}\right)\left(2-e^{u}\right)}
$$


moyennant la substitution $\log \left(1+\frac{1}{x}\right)=u$. Quand $x$ devient infinie ou devient zéro après avoir été positive, cette différence devient positive aussi. Enfin par la même substitution on a:

$$
x+x^{\frac{1}{2}}-\log ^{-1}\left(1+\frac{1}{x}\right)=\frac{\left(e^{x}-1\right)^{\frac{1}{4}}-\frac{u}{1 \cdot 2}-\frac{u^{2}}{1 \cdot 2 \cdot 3}-\cdots}{e^{u}-1}
$$

différence qui devient encore positive. Les trois fonctions en question devront être rangées dans l'ordre suivant:

$$
x \frac{x+1}{x-1}, \quad \log ^{-1}\left(1+\frac{1}{x}\right), \quad x+x^{\frac{1}{2}} .
$$

Art. II.

Théorèmes concernants le rapport entre les infinis des fonctions et de leurs dérivées.

Nous supposerons que les fonctions dont nous voulons comparer les infinis avec ceux de leurs dérivées, n'ont pas un nombre illimité de maxima et minima, ni même de points d'inflexion, car si le dernier cas avait lieu, c'est les dérivées qui pourraient avoir un nombre infini de maxima et minima et par conséquent ne pas être susceptibles d'une limite déterminée finie ou infinie.

1. Selon que l'on a $f(x) \succ_{\varphi} \succ_{(x)}$, en posant $f(x)=\Phi(x) \cdot f_{1}(x)$, on aura $f_{1}(\infty)={ }_{\infty}^{0}$. Si l'on a $f(x) \sim \Phi(x)$, et que l'on fasse $f(x)=M \varphi(x)+f_{1}(x)$ où $M=\lim \frac{f x}{\varphi(x)}$, on aura $f_{1}(x)<f(x)$.

Les relations $f(x) \stackrel{<}{\sim} \varphi(x)$ ne sont pas altérées quand on multiplie $f(x)$ $>$

et $\Phi(x)$ par des fonctions qui restent finies et déterminées et ne s'annulent pas pour $x=\infty$, par exemple par un constante d'une valeur quelconque, Annali di Matematica, tomo IV. 
ainsi les relations $\Phi(x) \succ_{\langle} x$ entrainent celles-ci : $e^{\varphi(x)}\left\langle e^{m x}, m\right.$ étant un nombre aussi petit et $M$ un nombre aussi grand qu'on voudra.

2. Lemme. On a $f^{\prime}(\infty)=\lim \frac{f(x)}{x}$, toutes les fois que $f^{\prime}(\infty)$ n'est pas indéterminée.

Démonstration. La formule $(b-a) f^{\prime}(\xi)=f(b)-f(a), a<\xi<b$, équivaut à une identité, pourvu que, dans le cas où $f^{\prime}(x)$ éprouve des changements brusques de valeur entre les limites $a$ et $b$ de $x$, on convienne de ne considérer $f^{\prime}(\xi)$ que comme une valeur moyenne entre les valeurs extrèmes de $f^{\prime}(x)$ dans l'intervalle $x=a, \ldots x=b$. Maintenant en écrivant cette formule ainsi qu'il suit:

$$
f^{\prime}(\xi)=\frac{1}{1-\frac{a}{b}}\left\{\frac{f(b)}{b}-\frac{f(a)}{b}\right\}, a<\xi<b,
$$

et en faisant d'abord $b=\infty$ puis $a=\infty$, on obtient le lemme en question.

3. Théorème. Aux trois cas $f(x) \underset{>}{\smile}$ répondent ceux-ci:

$$
f^{\prime}(\infty)=\underset{\infty}{0} \text { ni zéro ni infini. }
$$

Démonstration. En faisant $f(x)=x f_{1}(x)$ selon que $f(x) \succ_{<} x$, on a

$$
f_{1}(\infty)=\lim \frac{f(x)}{x}=\underset{0}{\infty}
$$

donc, d'après le lemme: $f^{\prime}(\infty)={ }_{0}^{\infty}$. Si l'on a $f(x) \cup x$, on fera

$$
f(x)=A x+f_{1}(x), \quad f^{\prime}(x)=A+f_{1}^{\prime}(x) .
$$

Or à cause de $f_{1}(x)<x$, et à cause de la première partie de cette démonstration, on a $f_{1}^{\prime}(\infty)=0$, donc $f^{\prime}(\infty)=A$.

4. Lemme. Soit $e^{M x}>f(x)>e^{m x}$, il sera permis de poser

$$
f(x)=f_{1}(x) e^{\mu x}
$$

où $M>\mu>m$, et où $f_{1}(x)$ satisfait à l'une ou à l'autre des conditions :

$$
f_{1}(x)<e^{\alpha x}, \quad \frac{1}{f(x)}<e^{\alpha x},
$$

$\alpha$ étant un nombre si petit que l'on voudra. 
Démonstration. Quand on a $e^{M x}>f(x)>e^{m x}$, on doit avoir $\log f(x) \cap x$, car en faisant $f(x)=e^{\phi(x)}$, on trouve

$$
\lim x\left\{\frac{\varphi(x)}{x}-M\right\}=-\infty, \quad \lim x\left\{m-\frac{\varphi(x)}{x}\right\}=-\infty
$$

donc $M>\lim \frac{\varphi(x)}{x}>m$, ainsi $\lim \frac{\varphi(x)}{x}$ est une quantité finie différente de zéro. Et à cause de $\Phi(x) \sim x$, en posant $\phi(x)=\mu x+\Phi_{1}(x)$, on aura $\Phi_{1}(x)<x$.

Maintenant si dans $f(x)=e^{\mu x+\varphi_{1}(x)}$ on fait $e^{\varphi_{1}(x)}=f_{1}(x)$, selon que $\phi_{1}(x)$ est positive ou négative, $f_{1}(x)$ satisfera à la première ou à la seconde condition du lemme.

En géneral, chaque fois qu'en posant $f(x)=e^{\varphi(x)}$, on aura $\varphi(x) \sim x$, on pourra indiquer deux nombres $\mu_{1}, \mu_{2}$ aussi rapprochés qu'on voudra et satisfaisants à l'inégalité infinitaire $e^{\mu_{1} x}>f(x)>e^{\mu_{2} x}$. Car en faisant $\phi(x)=$ $=\mu x+\phi_{1}(x), \mu_{1}>\mu>\mu_{2}$, les rapports de $e^{\mu_{1} x}$ à $f(x)$, et de $f(x)$ à $e^{\mu_{2} x}$ pourront s'écrire: $e^{x\left\{\mu_{1}-\mu-\frac{\left.\varphi_{1}(x)\right\}}{x}\right\}}, e^{x\left\{\mu-\mu_{2}+\frac{\varphi_{1}(x)}{x}\right\}}$, où l'on aura $\lim \frac{\varphi_{1}(x)}{x}=0$. Si au contraire il n'est pas possible d'indiquer un nombre $M$ suffisamment grand ou un nombre $m$ suffisamment petit, pour qu'on ait $e^{M x}>f(x)>e^{m x}$, on n'aura jamais $\phi(x) \sim x$.

5. Théoreme. Soit $f(x)$ une fonction qui a un infini plus grand que les puissances minimes $e^{x}$, et un infini moindre que les puissances le plus hautes de $e^{x}$, dans ce cas on aura toujours:

$$
f^{\prime}(x) \backsim f(x)
$$

Démonstration. En effet, en posant $f(x)=e^{\mu x+\varphi(x)}$, la différentiation logarithmique donne:

$$
\frac{f^{\prime}(x)}{f(x)}=\mu+\phi^{\prime}(x)
$$

Or à cause de $\Phi(x)<x$ et du théorème 3 , on a $\Phi^{\prime}(\infty)=0$, donc $\lim \frac{f(x)}{f(x)}=\mu$

6. Théorème inverse. Quand on n'a pas $e^{M x}>f(x)>e^{m x}$, M étant si grand et $m$ si petit qu'on voudra, on n'aura jamais $f^{\prime}(x) \sim f(x)$. 
En posant $f(x)=e^{p(x)}$, on n'aura pas $\phi(x) \sim x$, donc $\Phi^{\prime}(\infty)$ sera ou zéro ou infinie, et comme l'on a $\frac{f^{\prime}(x)}{f(x)}=\phi^{\prime}(x), \lim \frac{f(x)}{f(x)}$ sera de même ou zéro ou infini.

\section{Généralisation des théorèmes précédents, et classification des fonctions selon leurs types infinitaires.}

Ces théorèmes se généralisent facilement, vu que l'on peut remplacer l'exponentielle $e^{x}$ par une fonction quelconque $v$ devenant infinie pour $x=\infty$.

7. En effet, supposons que l'on ait $v^{M}>f(x)>v^{m}, M$ étant si grand et $m$ si petit qu'on voudra, on pourra écrire $f(x)=f_{1}(x) v^{\mu}, M>\mu>m$, et $f_{1}(x)$ satisfaisant à l'une ou à l'autre des conditions $f_{1}(x)<v^{\alpha}$, $\frac{1}{f_{1}(x)}<v^{\alpha}, \alpha$ étant de la dernière petitesse. C'est une consequence qui découle du lemme 4, si l'on fait $v=e^{*}, x=\psi(u)$.

8. De même si $v^{M}>f(x)>v^{m}$, on a toujours:

$$
\frac{f^{\prime}(x)}{\frac{d 1 v}{d x}} \cup f(x)
$$

Car en posant $v=e^{u}, x=\psi(u)$, d'après le théoreme 5 , on a

$$
\frac{d f(x)}{d u}=\frac{d f(x)}{d x} \frac{d x}{d u} \sim f(u)
$$

et de l'équation $u=1 v$, on tire $1=\frac{d l v}{d x} \cdot \frac{d x}{d u}$.

9. Enfin quand on n'a pas $v^{M}>f(x)>v^{\mu}$, on n'aura jamais

$$
\frac{f^{\prime}(x)}{\frac{d 1 v}{d x}} \sim f(x) \text {. }
$$

Car en faisant $e^{u}=v, x=\psi(u), f(x)=e^{\theta(u)}$, on n'aura pas $\theta(u) \sim u$, donc on aura $\frac{d \theta(u)}{d u}=\frac{d l f(x)}{d u}=\frac{f^{\prime}(x)}{f(x)} \cdot \frac{1}{\frac{d l v}{d x}}=$ à zéro, ou infinie.

On peut donner à ces théorèmes l'énoncé parfaitement symmétrique que voici. 
10. Soient $u$ et $v$ deux fonctions de $x$ devenant infinies pour $x=\infty$, si $\lim \frac{\frac{d l u}{d x}}{\frac{d l v}{d x}}$ n'est ni zéro ni infini, on pourra toujours donner à $u$ la forme $U v^{\prime \prime}, U^{ \pm 1}$ ayant un infini moindre qu'une puissance, quelque petite qu'elle soit, de $v$; et de même à $v$ on poura donner la forme $V u^{\mu}, V^{ \pm 1}$ ayant un infini moindre qu'une puissance de $u$. Réciproquement, quand on peut donner à $u$ ou à $v$ cette forme $U v^{\mu}$ ou $V u^{\mu}$, on aura toujours $\frac{d l u}{d x} \sim \frac{d l v}{d x}$.

Ces théorèmes conduisent à une espèce de classification des fonctions selon les infinis de leurs dérivées. Nommons la quantité $\frac{1}{\frac{d l v}{d x}}$ type infinitaire de la classe des fonctions dont l'infini n'atteint pas celui des puissances les plus hautes de $v$ et est supérieur a celui des puissances les plus basses de $v$ : a chaque type répondra une classe, mais la classe ne changera pas quand on multiplie le type par une constante, c'est à dire, quand on élève la fonction $v$, qui entre dans le type, à une puissance quelconque. Il y a donc moins de types, ainsi que cela doit etre, que de fonctions. Comme nous l'avons vu plus haut, l'unité est le type de toutes les fonctions $f(x)$, satisfaisantes à la condition $e^{M}>f(x)>e^{m x}$, ainsi $x$ sera le type de toutes les fonctions $x^{M}>f(x)>x^{\mu}$, pour lesquelles on aura donc $x f^{\prime}(x) \sim f(x), x l x$ sera le type des fonctions $1 x^{M}>f(x)>1: x^{m}$, pour lesquelles on aura $x l x f^{\prime}(x) \sim f(x)$, etc. etc.

\section{Quelques théorèmes concernant les types infinitaires des fonctions.}

Jetons un coup d'œil sur les trois séries suivantes, dont la première contient quelques fonctions rangées par ordre de grandeur de leur infinis, la seconde leurs dérivées, la troisième leurs types:

$$
\begin{array}{r}
e^{-e^{x}}, \quad e^{-x^{2}}, e^{-x}, \quad x^{-2}, x^{-1}, \quad x^{-\frac{1}{2}}, \log x^{-1}, \text { const, } 1 x, x^{\frac{1}{2}}, x, x^{2}, e^{x}, e^{x^{2}}, e^{e^{x}} \\
-e^{x} e^{-e^{x}},-2 x e^{-x^{2}},-e^{-x},-2 x^{-3},-x^{-2},-\frac{1}{2} x^{-\frac{3}{2}},-\frac{1 x^{-2}}{x}, 0, \quad 0 \quad \frac{1}{a}, \frac{1}{2} x^{-\frac{1}{2}}, 1,2 x, e^{x}, 2 x e^{x}, e^{x} e^{e} \\
-e^{-x}, \quad-\frac{1}{x},-1,-x,-x,-x,-x \ln , \quad,+x \ln , x, x, x, 1, \frac{1}{x}, \frac{1}{e^{-x}}
\end{array}
$$


En nous bornant à considérer les fonctions à droite de «const.», nous remarquons que $f(\infty)$ parcourant tous les infinis, des plus petits, immédiatement à droite de "const. ", jusqu'aux plus grands, $f^{\prime}(\infty)$ commence par s'évanouir, puis devient finie et différente de zéro, puis atteint un infini égal à celui de $f(x)$, et finalement son infini surpasse celui de $f(x)$.

La troisième série nous fait voir que les types réciproques peuvent atteindre les infinis les plus grands, mais les types directes ne peuvent vraisemblablement pas avoir un infini aussi grand qu'une puissance de $x$ supérieure à la première. Pour donner à ces conclusions plus de sureté, et pour faire en sorte qu'elles embrassent aussi les fonctions intermédiares à celles qui se trouvent dans la première série, notons ces théorèmes :

11. Théoréme. Soit $f^{\prime}(x)>\beta(x)$ et posons $f^{\prime}(x)=\lambda(x) f^{\prime}(x)$. Soit $f_{1}(x)>f(x)$, et posons $f_{1}^{\prime}(x)=\lambda_{1}(x) f_{1}(x)$. Je dis qu'on n'aura jamais $\lambda(x)>\lambda_{1}(x)$.

Démonstration. En faisant $f_{1}(x)=\varphi(x) f(x)$ on a :

$$
\frac{f_{1}^{\prime}(x)}{f_{1}(x)}=\frac{f^{\prime}(x)}{f(x)}+\frac{\varphi^{\prime}(x)}{\varphi(x)} \text {. }
$$

Au lieu de $f^{\prime}{ }_{1}(x), f^{\prime}(x)$, nous écrirons $\lambda_{1}(x) f_{1}(x), \lambda(x) f(x)$. Il vient:

$$
\lambda_{1}(x)=\lambda(x)+\frac{\varphi^{\prime}(x)}{\varphi(x)} \text {. }
$$

$\oint(x)$ devenant infinie pour $x=\infty, \lim \frac{\varphi^{\prime}(x)}{\varphi(x)}$ ne pourra pas être négative, donc etc.

12. Théorème. Soit $f^{\prime}(x)<f(x)$ et $f_{1}(x)<f(x)$,

$$
f^{\prime}(x)=\frac{f(x)}{\lambda(x)}, \quad f_{1}^{\prime}(x)=\frac{f_{1}^{\prime}(x)}{\lambda_{1}(x)}
$$

je dis que l'on n'aura jamais $\lambda(x)>\lambda_{1}(x)$.

Démonstration. Faisons $f_{1}(x)=\frac{f(x)}{\varphi(x)}$. Nous avons:

$$
\frac{f_{1}^{\prime}(x)}{f_{1}(x)}=\frac{f^{\prime}(x)}{f(x)}-\frac{\varphi^{\prime}(x)}{\varphi(x)}
$$

$\lim \frac{\varphi^{\prime}(x)}{\varphi(x)}$ étant positive, et $\lim \frac{f^{\prime}(x)}{f(x)}$ zéro, de

$$
\lim \frac{f_{1}(x)}{f_{1}(x)}+\lim \frac{\varphi^{\prime}(x)}{\varphi(x)}=0
$$


nous tirons d'abord $\lim \frac{f_{1}(x)}{f_{1}(x)}=0$. De plus, en introduisant les fonctions $\lambda$, nous trouvons:

donc etc.

$$
\lambda_{1}(x)=\lambda(x)+\lambda(x) \lambda_{1}(x) \frac{\varphi^{\prime}(x)}{\varphi(x)}
$$

Soit par exemple $f(x)=x^{2}, f_{1}(x)=x, \oint(x)=x, \lambda(x)=\frac{x}{2}, \lambda_{1}(x)=x$. L'équation $\lambda_{1}(x)=\lambda(x)+\lambda(x) \lambda_{1}(x) \cdot \frac{\varphi^{\prime}(x)}{\varphi(x)}$ donnera $\lambda_{1}(x)=x=\lambda(x)+\frac{x}{2}$. On a donc $\lambda_{1}(x) \sim \lambda(x)$. Soit en second lieu $f(x)=x, f_{1}(x)=\log x$, $\varphi(x)=\frac{x}{\log x}, \lambda(x)=x, \lambda_{1}(x)=x \log x$. L'équation entre les $\lambda$ donne

$$
\lambda_{1}(x)=\lambda(x)+x(\log x-1) .
$$

On a donc $\lambda_{1}(x)>\lambda(x)$. A ces théorèmes ajoutons encore celui-ci :

13. Soient $t_{1}, t_{2}, \ldots t_{r}$ les types de $r$ fonctions et soit $t_{r}$ le type dont la valeur directe ait l'infini le plus petit, ou la valeur reciproque l'infini le plus grand, $t_{r}$ sera le type du produit de ces $r$ fonctions.

En effet soient $f_{1}(x), f_{2}(x)$ deux fonctions avec les types $t_{1}, t_{2}$ et soit $t_{1}>t_{2}$. Dans

$$
\frac{d f_{1} f_{2}}{d x}=\frac{d f_{1}}{d x} f_{2}+\frac{d f_{2}}{d x} f_{1}
$$

on aura $\frac{d f_{1}}{d x} f_{2}<\frac{d f_{3}}{d x} f_{1}, t_{2} \cdot \frac{d f_{2}}{d x} \cdot f_{1} \sim f_{1} f_{2}$, donc $t_{2} \frac{d f_{1} f_{2}}{d x} \sim f_{1} f_{2}$.

De l'excès maximum de l'infini de la dérivée sur celui de la fonction.

La seconde des trois séries transcrites plus haut, savoir celle des dérivées des fonctions de la première série nous montre que, plus les infinis des fonctions sont grands, plus aussi les infinis des dérivées surpassent ceux des fonctions. Il est curieux d'établir de combien les infinis des dérivées peuvent surpasser ceux des fonctions. On obtiendra des éclaircissements suffisants sur ce sujet, au moyen des raisonnements suivants.

Quand une fonction de $x$ s'évanouit pour $x=\infty$ sans avoir un nombre infini de maxima et minima, sa dérivée s'évanouira aussi. Supposons donc 
que $f(x)$ devienne infinie pour $x=\infty, 1_{n}^{-v} f(x)$ devra s'évanouir pour $x=\infty$, ainsi la dérivée de $1_{n}{ }^{-"} f(x)$

$$
\frac{f^{\prime}(x)}{\left.f(x) \cdot 1_{1} f \cdot 1_{2}\right)} \frac{1 \cdots 1_{n}{ }^{1+\nu} f}{20}
$$

s'évanouira aussi, quelque grand que soit $n$ et quelque petit que soit $\nu>0$. Soit de plus $t(x) f^{\prime}(x) \sim f(x)$. L'infini de $f(x)$ étant supposé plus grand que celui d'une puissance de $e^{x}$, on aura $t(\infty)=0$.

14. Mais si l'on divise l'inégalité infinitaire $f^{\prime}(x)\left\langle f(x) \cdot l_{1} f \cdots l_{*}{ }^{4+\nu} f\right.$ par l'égalité $t(x) f^{\prime}(x) \sim f(x)$, on aura toujours:

$$
\frac{1}{t(x)}<l_{1} f \cdots l_{n}{ }^{1+\nu} f
$$

et à fortiori $\frac{1}{t(x)}\left\langle f^{\nu}, \nu\right.$ étant si petit qu'on voudra.

Quand $f(x)$ s'évanouira plus vite pour $x=\infty$ que $e^{-x}$, comme par exemple $f^{\prime}(x)=e^{-e^{x}}$, sa dérivée, dans l'exemple précedent $-e^{x} \cdot e^{-e^{x}}$, s'évanouira plus lentement que $f(x)$, le rapport $\frac{f^{\prime}(x)}{f(x)}$ aura une limite infinie. Mais si $f(\infty)=0$, $\lim l_{n}{ }^{-v} \frac{1}{f(x)}$ sera aussi égal à zéro par conséquent

$$
\frac{f^{\prime}(x)}{f \mathrm{~L}_{1} \frac{1}{f} \mathrm{~L}_{2} \frac{1}{f} \cdots 1_{n}^{1+\nu} \frac{1}{f}}
$$

s'évanouira aussi, quelque grand que soit $n$ et quelque petit que soit $\nu$, pourvu qu'on ait $\nu>0$.

15. Si donc on fait $l(x) f^{\prime}(x) \curvearrowright f(x)$, on obtiendra de nouveau

$$
\left.\frac{1}{i(x)}<l_{1} \frac{1}{f} \cdots\right]_{n}{ }^{1+\nu} \frac{1}{\ddot{f}}
$$

et à fortiori $\frac{1}{t(x)}<\frac{1}{f(x)^{2}}$, inégalité qui s'obtient directement en songeant que $f(x)^{\prime}$ et sa dérivée doivent s'évanouir pour $x=\infty$, donc on a $f^{\prime}(x)\left\langle f(x)^{1-\nu}\right.$, inégalité qu'il faut diviser par $t(x) f^{\prime}(x) \sim f(x)$.

De cette manière nous avons déterminé l'infini que ne saurait surpasser le rapport de la dérivée à la fonction chez les fonctions aux infinis les plus grands, ou bien aux zéros les plus petits. 
Sur les infinis des fonctions pour des valeurs finies de la variable.

Il est facile de réduire les infinis des fonctions pour une valeur finie de $x$, par exemple pour $x=0$, à ceux que nous avons considérés jusqu'ici. En faisant $\frac{1}{x}=x_{1}, f(x)=F\left(x_{1}\right)$ on aura $f^{\prime}(x)=-x_{1}^{2} F^{\prime}\left(x_{1}\right)$, et comme $x_{1}$ s'évanouit pour $x=\infty$, on peut exprimer chaque théorème concernant $f(\infty)$ et $f^{\prime}(\infty)$ comme théorème touchant $F(0)$ et $F^{\prime}(0)$. Nous finissons par noter encore une proposition qui se rapporte aux infinis de $f(x)$ et $f^{\prime}(x)$ pour $x=0$, et dont la démonstration directe est très simple.

16. Quand $f(x)$ devient infinie pour $x=0$, on a

$$
f^{\prime}(x)>f(x) 1_{1} f \ldots 1_{n}^{1-x} f
$$

( $\nu$ autant petit qu'on voudra) et quand $f(x)$ s'évanouit pour $x=0$, on a

$$
f^{\prime}(x)>f(x) l_{1} \frac{1}{f} \cdots l_{n}{ }^{1-\nu} \frac{1}{f}
$$

En effet, si la fonction $f(x)$ devient infinie pour $x=0$, sans avoir un nombre infini de maxima et minima, la dérivée doit devenir infinie aussi, parce que la tangente asymptotique est verticale. Donc les dérivées de $1_{n}{ }^{\prime} f^{\prime}$ pour $f(0)=\infty$ et de $1_{n}{ }^{\prime} \frac{1}{f}$ pour $f(0)=0$ seront infinies pour $x=0$.

Fine deL Tomo IV. ${ }^{\circ}$ (SerIe II. ${ }^{a}$ ) 